Disponible en ligne : www.cahiersagricultures.fr

\title{
L'agriculture au Sahara du sud-ouest algérien : entre développement agricole moderne et permanences de l'agriculture oasienne traditionnelle
}

\author{
Mohamed Hadeid*, Sid Ahmed Bellal, Tarik Ghodbani et Ouassini Dari \\ Département de Géographie et de l'Aménagement du Territoire, Laboratoire EGEAT, Université d'Oran 2, Oran, Algérie
}

\begin{abstract}
Résumé - L'agriculture oasienne reflète l'ingéniosité d'une société ayant pu vaincre un milieu hostile et défavorable au peuplement. En Algérie, cette agriculture traditionnelle a été bouleversée par l'introduction des emplois non-agricoles, qui ont perturbé la stratification sociale établie. Une nouvelle politique de mise en valeur agricole a été instaurée depuis 1983 afin de remplacer l'agriculture traditionnelle considérée comme peu rentable et ne pouvant faire face à de nouveaux problèmes (manque de main-d'oeuvre, déclin des systèmes hydrauliques à foggaras...) et de lancer les régions arides dans une phase de production céréalière en niant l'organisation sociale féodale antérieure. Depuis, l'intérêt de l'État pour cette agriculture moderne s'est manifesté essentiellement par différentes subventions. Tout cela s'est traduit par un recul accéléré de l'agriculture oasienne au profit de l'emploi dans les services et l'administration, ou dans les nouvelles exploitations créées dans les nouvelles zones de mise en valeur agricoles. Cependant, plus récemment, certaines transformations commencent à voir le jour dans des oasis auparavant en difficulté. Dans une période où l'emploi tertiaire est saturé et où les aides de l'État s'affaiblissent progressivement, les oasiens ont décidé de s'adapter en tentant de retourner au travail de la terre dans les palmeraies délaissées, tout en s'orientant vers des cultures rémunératrices. On observe ainsi non pas une concurrence de l'agriculture moderne avec l'agriculture oasienne, mais le développement d'une autre vision, fondée sur la recherche du profit, aussi bien dans l'agriculture saharienne considérée comme moderne que dans celle considérée comme traditionnelle.
\end{abstract}

Mots clés : agriculture oasienne / État / foggaras / mise en valeur agricole / palmeraies

\begin{abstract}
Agriculture in the Algerian south-west Sahara: between modern agricultural development and traditional oasis agriculture permanencies. Oasis agriculture reflects the ingenuity of a society able to overcome hostile and unfavorable conditions for settlement. In Algeria, this traditional agriculture was severely affected by the introduction of non-agricultural employment, thus disrupting the social configuration. The new State policy based on agricultural development has been established since 1983 to replace this traditional agriculture, considered less profitable and a source of many problems (lack of workforce, declining of traditional foggara irrigation systems...), and to develop cereal production in the region, negating any previous social organization. Since then, the interest of the state focused on modern agriculture through various subsidies. This attitude has further weakened the interest for oasis agriculture in favor of employment in services and administration, or in the new agricultural development schemes. But recently, some changes have been observed, especially in oases formerly declining. Indeed, at a time when the service sector is overcrowded and State subsidies are gradually decreasing, the oasis dwellers have decided to adapt themselves by trying to return to work the land in the abandoned palm groves. The current period is thus characterized, not by a competition of modern versus traditional oasis agriculture, but by a development of a new vision of a liberal nature. These new trends in traditional and modern Saharan agriculture are in fact an adaptation to the country's political and economic context.
\end{abstract}

Keywords: oasis agriculture / State / foggaras / agricultural development / palm groves

\footnotetext{
*Auteur de correspondance : hadeid009@yahoo.fr
} 


\section{Introduction}

Reflétant l'ingéniosité d'une société ayant su surmonter un milieu hostile et défavorable au peuplement, l'agriculture oasienne est un élément fondamental de l'espace saharien (Bisson, 2003). Elle représentait une activité indispensable pour ravitailler à la fois les populations oasiennes et les caravaniers qui sillonnaient le Sahara (Côte, 2002). L'organisation économique ancestrale a changé au fil du temps, puisque l'apparition des activités non-agricoles a engendré une diversification des emplois et une régression du poids de l'agriculture oasienne, qui était fondée sur une stratification sociale bien marquée (Marouf, 1980). En effet, par l'introduction d'emplois, en particulier dans le tertiaire, mais aussi le secondaire dans la région du Sud-Est algérien et plus récemment dans celle du Sud-Ouest, l'État algérien indépendant a fortement perturbé cette agriculture traditionnelle. Du fait du bouleversement de la stratification sociale établie depuis des siècles, l'agriculture a perdu du terrain par rapport aux nouvelles activités. Depuis 1983, la politique nationale, fondée sur la mise en valeur agricole des steppes et du Sahara, vise à remplacer cette agriculture traditionnelle considérée en difficulté et peu rentable, par la production céréalière, en niant l'organisation sociale féodale antérieure. Mais, malgré les subventions généreuses de l'État, cette nouvelle politique n'a pas pu atteindre les objectifs fixés au départ (Otmane, 2010; Hadeid, 2011). Durant ces dernières années, marquées par un désengagement progressif de l'État, de nouvelles dynamiques agricoles sont apparues, aussi bien dans le secteur agricole moderne que dans le secteur traditionnel. Motivée par le profit, voire la spéculation, cette nouvelle tendance de l'agriculture oasienne semble lui donner un nouveau souffle dans une période où l'État ne dispose plus des ressources financières pour maintenir le soutien assuré auparavant. Les études effectuées jusqu'à présent sur l'agriculture en milieu oasien ont tendance à «pointer du doigt» la mise en valeur moderne comme l'un des facteurs les plus importants ayant conduit au recul de l'agriculture traditionnelle. L'objectif de cet article est de nuancer ce constat et d'actualiser les connaissances sur les nouvelles dynamiques rurales qui caractérisent l'espace oasien algérien d'une manière générale. Il s'agit également d'apporter un nouveau regard sur la dynamique actuelle qui se traduit par l'émergence de liens nouveaux entre le monde oasien traditionnel et les nouvelles technologies agricoles. Les dernières publications abordent la mobilité des jeunes ruraux (Naouri et al., 2015), les nouvelles technologies sociales (Ould Rebai et al., 2017) ou l'émancipation des jeunes oasiens (Hamamouche et al., 2015). Toutes ces recherches montrent que les deux systèmes agricoles (traditionnel et moderne) ne fonctionnent plus d'une manière isolée. La discontinuité spatiale causée par l'isolement géographique du Sahara, couplée à la stratification sociale, s'opposent de moins en moins aux échanges de savoir-faire et au partage des ressources naturelles. Les travaux de Bellal et al. (2016), de Daoudi et al. (2017) ou d'Amichi et al. (2015) montrent l'ouverture du système hydroagricole oasien à l'innovation. Les nouveaux savoir-faire agricoles véhiculés par la main-d'œuvre venue du Nord, et la forte volonté d'adaptation exprimée par les autochtones, associés à l'arrivée de main-d'œuvre subsaharienne, favorisent ainsi un développement remarquable de pratiques et de techniques hybrides, qui représentent des défis socio-économiques mais aussi environnementaux.

\section{Méthodologie de recherche et cadre d'étude}

S'insérant dans un cadre de géographie rurale, cette recherche s'appuie sur les nombreuses recherches effectuées dans la région d'Adrar sur le système oasien et son évolution. Elle est enrichie par une vingtaine d'enquêtes directes auprès des agriculteurs évoluant dans les exploitations de mise en valeur agricole situées dans la zone d'Aougrout et une quarantaine d'enquêtes auprès des paysans vivant dans les palmeraies de Tittaf et de Tilouline, alimentées par des foggaras (Fig. 1). La foggara (équivalent algérien du qanat moyen-oriental) est un moyen souterrain de captage de l'eau des nappes par des galeries drainantes, qui amène cette eau par gravité depuis le plateau de Tadmaït jusqu'aux terrains à irriguer. Ces galeries sont jalonnées par des puits verticaux destinés à permettre leur accès et leur entretien.

Ces enquêtes se sont intéressées aux exploitants (origine, statut social, motivation, contraintes...) et à leurs exploitations (surface, production, mode d'irrigation, statut foncier, système agricole, commercialisation...). Le choix de ces zones a été fait sur la base d'observations de terrain sur plusieurs années dans une centaine d'oasis de la wilaya (département) d'Adrar. D'autres entretiens ont concerné une dizaine d'acteurs institutionnels (collectivités locales, services agricoles et de l'hydraulique) afin de récolter des données sur les exploitations existantes, mais aussi pour recueillir leur avis sur la nouvelle dynamique de l'agriculture oasienne.

La zone d'étude concerne la wilaya d'Adrar, située dans le sud-ouest algérien. Cette wilaya s'étend sur près de $430000 \mathrm{~km}^{2}$, soit $18 \%$ du territoire national (Fig. 1). Elle abrite plus de 400000 habitants vivant dans près de 300 oasis et répartis dans 4 sous-régions (Gourara, Touat, Tidikelt, Tanezrouft). Les oasis choisies ici pour leur dynamisme agricole symbolisent bien les nouveaux enjeux du développement de l'agriculture oasienne.

\section{Le recul de l'agriculture oasienne durant la période post-Indépendance: causes et effets sur l'espace et la société}

L'oasis n'est pas seulement un «lieu habité isolé dans un environnement aride ou plus généralement hostile dont la localisation est liée à la possibilité d'exploiter une ressource, notamment l'eau, pour la pratique de l'agriculture irriguée » comme le précisent Lévy et Lussault, (2003, p. 671), mais elle a également servi de relais au commerce caravanier. L'agriculture oasienne a contribué largement à satisfaire les besoins alimentaires des populations (Bisson, 2003). Cependant, «l'installation de la colonisation au début du $\mathrm{xx}^{\mathrm{e}}$ siècle dans le pays des foggaras s'est matérialisée par une présence physique de l'administration coloniale, par la mise en place d'une circonscription administrative, l'introduction de l'impôt et par le contrôle des voies commerciales transsahariennes » (Otmane, 2010, p. 88). Ces actions ont conduit à l'affaiblissement du réseau d'échanges, privant ainsi les oasis d'une main-d'œuvre destinée habituellement au creusement et à l'entretien des foggaras (Capot-Rey, 1953). Cet élément a été capital dans le recul de l'activité agricole. Après l'Indépen- 


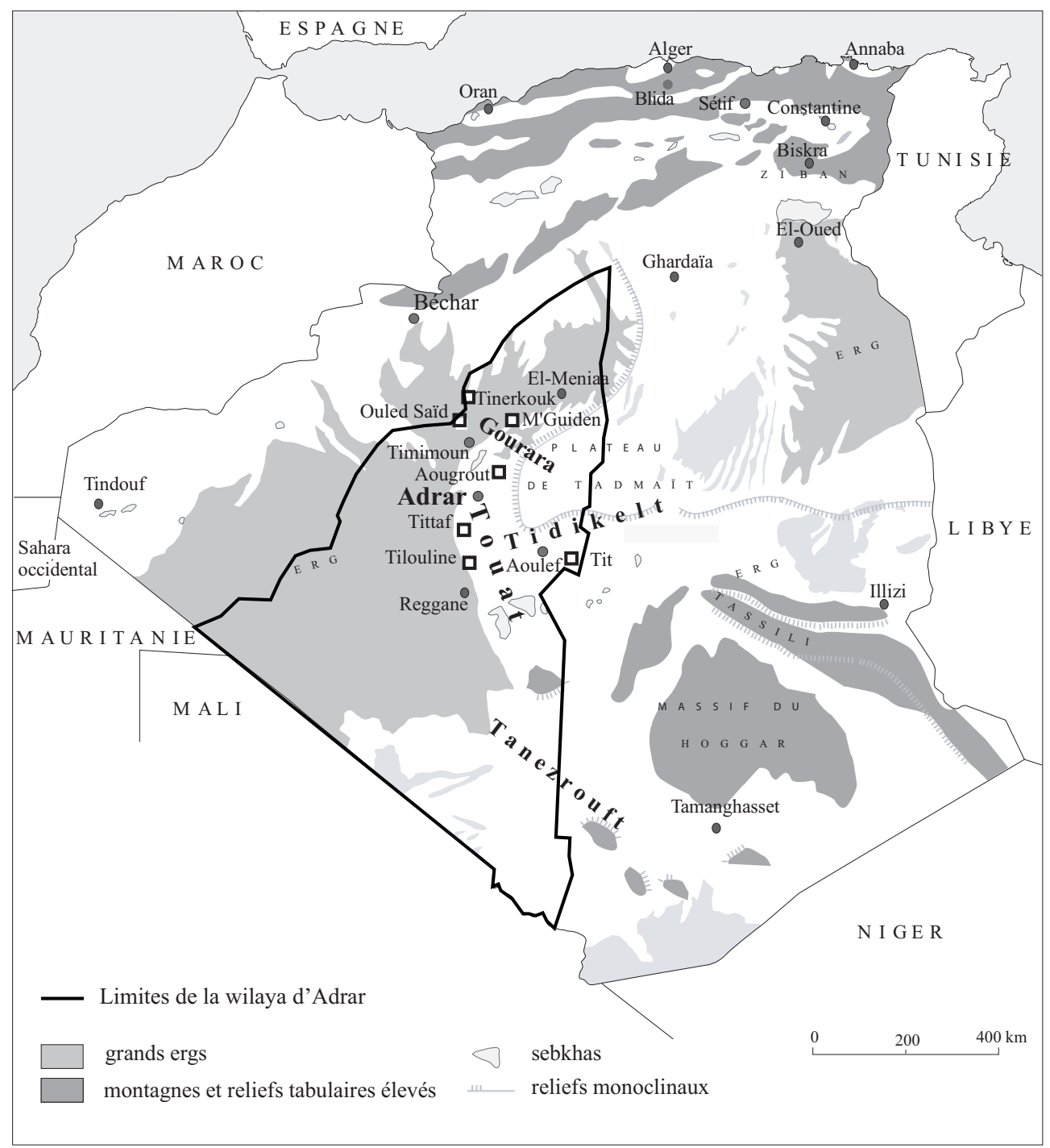

Fig. 1. Carte de localisation.

Fig. 1. Situation map.

dance, la nouvelle structuration administrative instaurée par l'État est également responsable de nombreux changements dans la manière de gérer ces espaces. L'organisation sociale commence à être sérieusement modifiée. En effet, l'assemblée communale s'est substituée progressivement aux prérogatives de la djemaâ, le conseil représentant la collectivité. Cela a permis aux fils des Harratines (Hommes de couleur qui travaillent la terre: harth signifie labour) de se libérer d'une discrimination sociale et d'accéder aux assemblées populaires communales et à d'autres postes de responsabilité dans les services de l'État. Ce bouleversement a pris une nouvelle envergure après la mise en œuvre de la Révolution agraire durant les années soixante-dix. Les principes de cette révolution sont clairs en ce qui concerne "l'élimination de toute forme d'exploitation du travail d'autrui en rétablissant dans l'agriculture des rapports directs de travail, basés sur le principe : la terre appartient à ceux qui la travaillent " (Charte de la révolution agraire et Ordonnance 71-73 du 8 novembre 1971 portant Révolution agraire, Journal officiel de la République algérienne $\mathrm{n}^{\circ} 30$ novembre 1971). Propriétaires fonciers des palmeraies et de l'eau de la foggara, les Chorfa (populations arabes) et les Mrabtine (hommes de religion d'origine berbère) des ksour (villages traditionnels) de la région d'Adrar, expliquent que cette réforme agraire est à l'origine de la refonte des rapports sociaux au sein de la société oasienne. Depuis, "ces propriétaires fonciers qui ne travaillaient pas la terre se sont trouvés, en quelques années, coupés de "leurs Harratines " qui possédaient à la fois le savoir-faire dans l'agriculture, et dans le forage et l'entretien permanent des galeries de foggaras » (Bendjelid et al., 1999, p. 46). Les entretiens effectués avec les Harratines nous ont confirmé que cette réforme a contribué à les « libérer» et qu'ils ont pu, par la suite, acquérir des terrains pour les cultiver et même obtenir des logements dans le cadre des villages socialistes agricoles.

Associé aux autres actions étatiques, ce bouleversement des structures sociales anciennes voulu par l'État a contribué d'une manière décisive au recul de l'agriculture oasienne. Toutefois, d'autres facteurs directs et indirects, ayant concouru à la régression de cette agriculture traditionnelle, méritent d'être signalés ici. En premier lieu, la question de l'eau est 
centrale. Les auteurs travaillant sur ce type d'espaces (Marouf, 1980 ; Dubost, 1991; Côte, 2002; Bisson, 2003) signalent l'importance du système hydraulique que constitue la foggara dans le maintien de l'agriculture oasienne: "la période postérieure à l'Indépendance a été défavorable à l'entretien des foggaras, en particulier à leur financement par le pouvoir central socialisant, ceci en raison à la fois de l'existence de rapports sociaux de servilité dans les ksour et d'une sorte de déconsidération de la foggara en tant que technique traditionnelle reflétant ces mêmes rapports. Ce contentieux a longuement pesé sur la pérennité de la méfiance qui existe entre les propriétaires fonciers et le pouvoir politique central » (Bendjelid et al., 1999, p. 46). La problématique de la diminution des débits des foggaras est le résultat de plusieurs processus, sociaux et techniques. Avec la croissance de la population oasienne, les besoins en eau ont augmenté pour pouvoir satisfaire à la fois les demandes pour l'eau potable et pour l'irrigation. La multiplication des forages, utilisant des méthodes modernes de pompage des eaux souterraines, a contribué au rabattement progressif de la nappe. Selon l'ANRH (Agence nationale des ressources hydrauliques), le taux des foggaras taries en 2004 a été évalué à plus de $35 \%$; en 2011, il a atteint près de $45 \%$, pour arriver à près de $53 \%$ en 2014 .

L'autre facteur est d'ordre environnemental. L'ensablement est un phénomène qui existe depuis toujours au Sahara, mais la paysannerie locale a tenté de le limiter en utilisant des matériaux produits localement (palmes sèches, argile...). Cependant, le manque d'entretien des dunes artificielles, les « afreg » conçues pour abriter le ksar et la palmeraie, est l'une des principales causes de leur ensablement, qui est un frein à l'activité agricole.

De même, l'augmentation des surfaces affectées par la salinité en raison de la baisse du niveau hypsométrique de la nappe et de l'augmentation des quantités d'eaux usées rejetées sans traitement préalable, conduit à la diminution des surfaces agricoles dans presque toutes les palmeraies de la région.

Cependant, le recul de l'agriculture oasienne ne peut être expliqué uniquement par ces facteurs physiques (assèchement des foggaras, ensablement, salinité...). Les actions de l'État ont aussi fortement contribué à la régression agricole : la révolution agraire a été suivie par la promotion de la région d'Adrar en wilaya en 1975, puis par le lancement de la politique de mise en valeur via la loi portant Accès à la propriété foncière agricole (APFA) en 1983. Ces actions ont perturbé non seulement le secteur agricole, mais aussi la société oasienne toute entière: bouleversement de la structure sociale, multiplication des emplois dans les secteurs non-agricoles, scolarisation, orientation vers l'agriculture moderne, diversification de l'économie, développement d'activités industrielles après la découverte des gisements de pétrole et de gaz.

\section{Les conséquences de la politique agricole: régression de l'agriculture oasienne et ruée vers la mise en valeur moderne}

\subsection{Introduction de l'agriculture moderne en milieu oasien: de la phœniciculture à la céréaliculture}

L'agriculture moderne a été introduite en milieu saharien par la promulgation de la loi portant Accès à la propriété

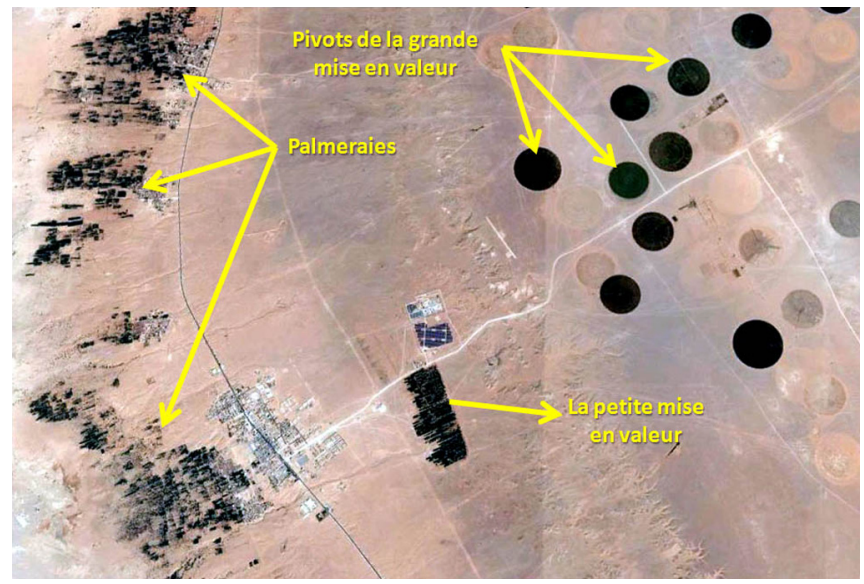

Fig. 2. Palmeraies traditionnelles et périmètres modernes coexistent. Source: image Google-Earth, 2013.

Fig. 2. Traditional palm groves and modern schemes coexisting.

foncière agricole en 1983. Cette loi a pour objet d'attribuer, à tout individu, des terres agricoles, ou à vocation agricole, pour les mettre en valeur par des travaux d'aménagement, de défrichement, de mobilisation de l'eau, d'équipement et de plantation... Cependant, l'acquéreur ne devient propriétaire qu'après la réalisation du travail de mise en valeur et au bout de cinq années. Cette nouvelle loi a été motivée par le constat que les politiques agricoles antérieures n'avaient pas réussi à limiter la dépendance alimentaire du pays dans un contexte caractérisé par une forte croissance démographique (Bessaoud, 2008). L'État s'est alors engagé dans une vaste opération de mise en valeur des zones arides et semi-arides en s'inspirant des expériences plus ou moins réussies des États-Unis, de l'Arabie Saoudite et de la Libye : "le déséquilibre alimentaire de l'Algérie est à l'origine des projets de mise en valeur des terres sahariennes, promues au rang d'un nouvel El Dorado, l'idée...étant de produire du blé» (Dubost, 1991, p. 311) pour faire de l'agriculture saharienne la réserve alimentaire de l'Algérie (Bessaoud, 2008), chose que l'agriculture du nord du pays n'avait pas réussi à faire.

Les périmètres de mise en valeur moderne créés dans le cadre de l'APFA sont localisés à proximité des palmeraies traditionnelles (Côte, 2002). Par conséquent, le paysage rural oasien a connu des changements avec l'irruption de nouvelles formes d'exploitation et d'appropriation de la terre (Fig. 2), qu'il s'agisse de la «petite mise en valeur» paysanne ou de la «grande mise en valeur» capitaliste.

Alors que le système agricole traditionnel fonctionnait sur la base d'une exploitation limitée des nappes souterraines par les foggaras, le nouveau système est complètement différent puisqu'il est basé sur le pompage de l'eau par forage. Cette mobilisation sans limite de l'eau conduit à une multiplication des aires d'irrigation. Cette vaste opération technique visait dès le départ l'aménagement de 100000 ha. Selon les données du CENEAP (Centre national d'études et d'analyses pour la population et le développement), entre 1986 et 1989, l'État a mobilisé près de 350 millions de dinars algériens $(2600000 €)$ pour cette opération de mise en valeur agricole (CENEAP, 1990). " Mais le constat établi dès 1992 a fait ressortir que sur les 100000 ha proposés à l'aménagement, 60000 ha ont été 
mis en valeur et seulement 45000 ont été réellement mis en culture » (Sahli, 1997, p 288).

L'apport de l'État est indéniable: l'opération de mise en valeur agricole via l'APFA a permis d'équiper les nouvelles exploitations en forages, énergie électrique, chemins ruraux... Les exploitants ont même pu accéder à des crédits bancaires. Cet apport s'est encore amplifié après le lancement du PNDA (Plan national de développement de l'agriculture) en 2000. Les exploitants des nouveaux périmètres de mise en valeur ont de nouveau pu bénéficier du financement d'opérations de forage, de réhabilitation d'infrastructures hydrauliques, d'équipement hydro-agricole (motopompes, bassins de stockage, réseaux de goutte à goutte) et de plantation de palmiers. L'APFA visait avant tout à augmenter la production céréalière. Le PNDA, lui, a relancé la production agricole en prenant en compte les potentialités agricoles locales, d'où le choix de la phœniciculture pour le Sahara. Le PNDA a suscité l'engouement de la population oasienne en raison de la générosité de ses financements. Cependant, le bilan est en deçà des aspirations. Sur les 60000 ha attribués dans le cadre de la mise en valeur agricole dans la wilaya d'Adrar, seul un tiers est réellement travaillé (DPAT d'Adrar, 2012). L'apport financier du PNDA a induit des investissements agricoles souvent délaissés par la suite. Cette non-mise en valeur concerne en majorité des commerçants, des entrepreneurs ou des agents de l'administration qui n'ont aucun lien avec l'activité agricole, et qui ont utilisé l'apport financier pour d'autres activités (Otmane, 2010; Hadeid, 2011).

Cependant, cette politique de mise en valeur agricole a conduit l'espace oasien à s'ouvrir sur les autres régions du pays, permettant à beaucoup d'investisseurs du Nord de s'installer. Conjuguée à d'autres formes de mobilité dans l'administration et le commerce particulièrement, elle est ainsi à l'origine d'une recomposition de la population oasienne. De même, l'accès aux emplois non agricoles, devenu une nécessité pour les oasiens pour diversifier et accroître leurs revenus, ainsi que l'accès à la mise en valeur agricole moderne, ont conduit la société oasienne à de réels changements, tant dans les comportements des individus que dans leurs modes de vie.

\subsection{Après le recul de l'État: apparition de nouveaux agriculteurs et revivification de l'agriculture oasienne}

\subsubsection{Les nouveaux agriculteurs: vers le maraîchage}

Le bilan mitigé de la politique de mise en valeur agricole, notamment après l'application du PNDA, ainsi que la diminution des ressources financières du fait de la diminution des prix du pétrole, ont conduit l'État à baisser le taux des subventions accordées à l'équipement des exploitations agricoles et ce, d'une façon progressive jusqu'à leur suppression totale en 2007. Ce retrait de l'État a été interprété comme un autre facteur de déclin de l'activité agricole en milieu oasien. En réalité, ce désengagement a aussi des effets positifs : cela a conduit les agriculteurs de la grande mise en valeur agricole à investir davantage eux-mêmes dans leurs exploitations. Cet investissement se traduit sur le terrain par la multiplication des serres destinées aux cultures maraîchères, comme c'est le cas à Aougrout (Fig. 3a et b), en particulier pour le concombre ( $78 \%$ des surfaces maraîchères), pour lequel il existe un important marché.
En louant les terres appartenant aux agriculteurs autochtones, qui se sont désengagés après la diminution du montant des subventions de l'État, les agriculteurs originaires de Sétif ont en quelques années généralisé la culture sous serre dans cette région (Fig. $4 \mathrm{a}$ et $\mathrm{b}$ ).

Des alliances ont commencé à prendre forme entre les nouveaux propriétaires du foncier (dont $60 \%$ portent des noms d'origine hartani et ont donc connu une forte ascension sociale) et les nouveaux investisseurs (venus du Nord) depuis le début des années 2000 (Ouendeno et al., 2015). L'arrivée des gens de Sétif n'est pas un effet du hasard, puisqu'ils étaient déjà en relation directe avec les commerçants de la région pour le transport des produits alimentaires. Abdelkader, jeune investisseur qui a loué une quarantaine d'hectares auprès d'un autochtone, explique: "J'ai une grande expérience dans les cultures sous serre, j'ai déjà une vingtaine de serres à Ä̈nOulmene, c'est une activité très rentable, ici l'eau est disponible et les récoltes sont vendues en avance par rapport au Nord. Je travaille ici de septembre à mars avant de retourner chez moi avec l'arrivée des premières vagues de chaleur».

Ces dernières années, le nombre de serres dans cette commune n'a cessé de se multiplier. On en compte actuellement plus de 2200 dans la commune d'Aougrout selon les données de la direction des services agricoles. Cet engouement pour les cultures maraîchères s'est accompagné d'un intérêt pour d'autres cultures (céréales, maïs), ainsi que pour l'élevage ovin et bovin. Néanmoins, il faut signaler le caractère minier et non durable de l'usage des ressources naturelles. D'une part, les quantités d'eau fossile utilisées dans l'irrigation sont importantes. D'autre part, la dégradation du sol causée par la monoculture et l'absence de périodes de jachère et de cycles de lessivage des sels minéraux, entraîne un déplacement des serres et des pivots tous les deux ou trois ans. Selon les techniciens de la subdivision des services agricoles d'Aougrout, l'usage incontrôlé des fertilisants représente un risque de contamination des aquifères. Sur le plan socioéconomique, cette dynamique agricole ne profite que peu à l'économie de la commune et encore moins à celle de la wilaya d'Adrar. Mis à part les rentrées directes en argent pour la location des terres, qui vont aux propriétaires, les bénéfices ne retournent pas à la population locale. Les oasis traditionnelles limitrophes ne participent pas à cette dynamique exogène: même le fumier est importé de l'est du pays, alors que la biomasse fournie par les palmeraies et l'élevage traditionnel pourrait alimenter les nouvelles exploitations en fertilisants. Hafid, un agriculteur, déclare que la forte demande de fertilisants l'oblige à importer du fumier: "Je ramène le fumier des poulaillers de Sétif avec ma propre camionnette, le fumier me pose des problèmes d'hygiène mais je ne peux pas attendre la fin du cycle de la fermentation, heureusement que les pesticides sont là pour nous sauver».

La main-d'œuvre, jugée insuffisante dans la région, est fournie par les migrations trans-sahariennes. Ainsi, la maind'œuvre sub-saharienne se concentre dans les périmètres de mise en valeur agricole d'Adrar. Habitant les dortoirs situés à l'intérieur de l'exploitation, elle est utilisée d'une manière informelle dans les travaux de gardiennage, nettoyage et récolte. Certains propriétaires des jardins situés dans les palmeraies traditionnelles l'utilisent aussi occasionnellement pour le nettoyage des palmiers. L'expansion du secteur tertiaire et d'activités industrielles pétrolières ont dissuadé les ouvriers 

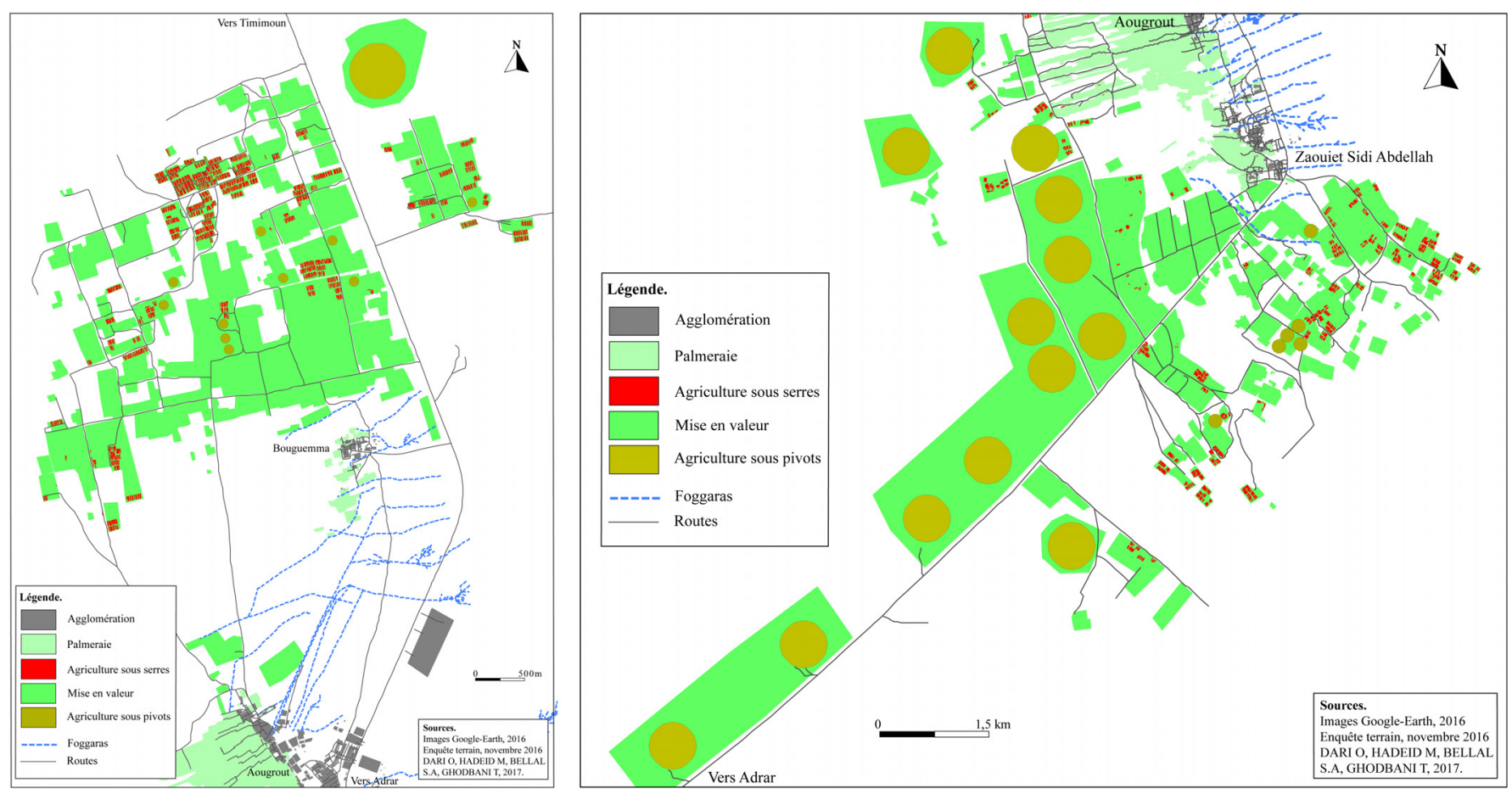

Fig. 3. a et b. Occupation du sol dans les deux périmètres irrigués d'Aougrout (Wilaya d'Adrar).

Fig. 3. Land use in two irrigated schemes in Aougrout (Adrar Wilaya).
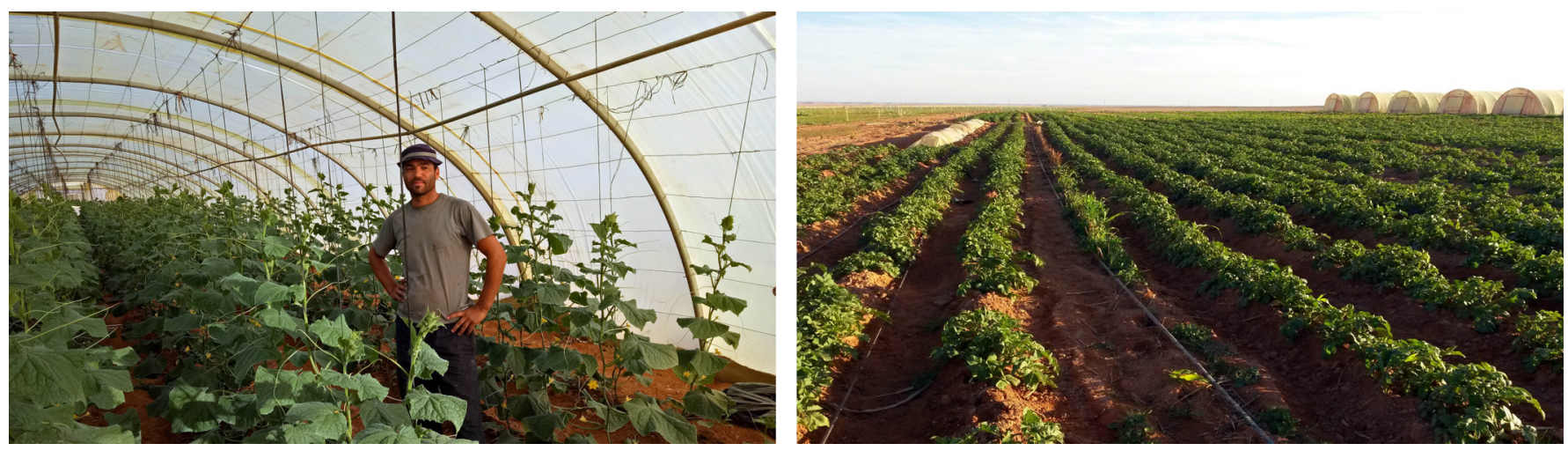

Fig. 4. $a$ et b. Exploitation agricole moderne à Aougrout dirigée par un originaire de Sétif. Cliché auteur, 12/2015.

Fig. 4. Modern farm in Aougrout operated by a man from Setif.

agricoles de rester dans ce secteur très faiblement rémunéré. Les jeunes Algériens choisissent des secteurs qui offrent un travail moins pénible et un revenu plus régulier que le travail de la terre, considéré comme socialement inférieur et réservé aux descendants des Harratines. Cette fuite de la maind'œuvre agricole est lourde de conséquences à la fois pour l'agriculture moderne et l'agriculture traditionnelle.

\subsubsection{La nouvelle tendance de l'agriculture oasienne: les cultures spéculatives}

Une bonne partie des palmeraies de la région d'Adrar ont connu une forte modification de l'occupation du sol: en plus des cultures maraîchères (pomme de terre, oignon, betterave, salade), les oasiens s'adonnent à la culture de la tomate industrielle. La réouverture de l'usine de Reggane, qui a établi des contrats de vente au champ avec les agriculteurs, en témoigne. Construite dans les années soixante-dix, cette usine n'avait jamais pu fonctionner correctement faute de production suffisante et régulière dans le Touat.

Plus récemment, la culture du tabac a pris de l'ampleur dans plusieurs palmeraies du Touat. Cette culture a vu le jour ces dernières années, quand la région a connu une forte immigration sub-saharienne. Ce produit est très demandé pour une utilisation locale et pour l'exportation vers les pays du Sahel (tabac à mâcher, « chemma »). Le tabac semi-traité peut se vendre de 600 à $1000 \mathrm{DA} / \mathrm{kg}(5$ à $8 €)$. À Tilouline et Tittaf, cette culture occupe près du tiers des surfaces des palmeraies. "Les feuilles de tabac séchées sont commercialisées à l'état brut... La contribution de la culture de tabac au revenu 


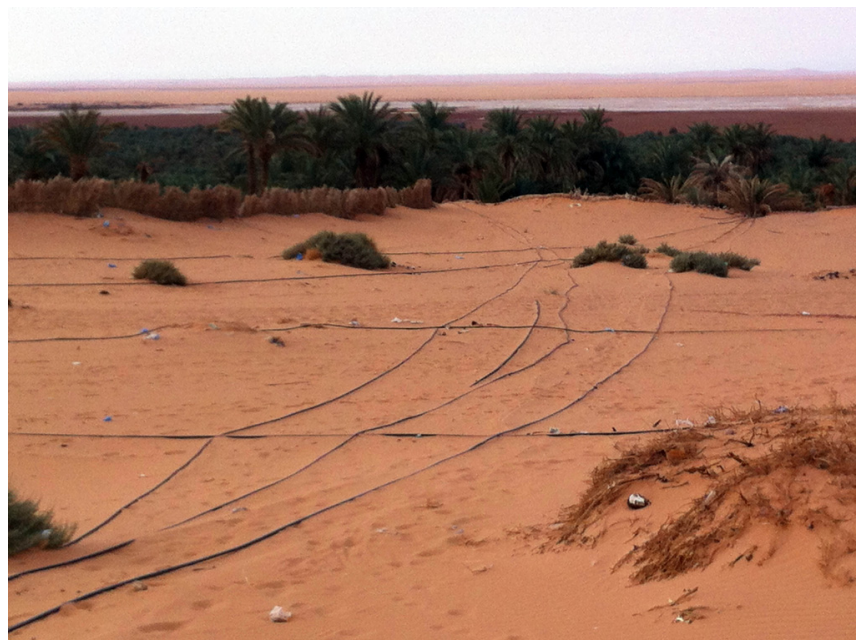

Fig. 5. Réseau d'eau potable piraté pour irriguer les jardins à Tilouline (commune d'In-Zeghmir). Cliché auteur, 12/2013.

Fig. 5. Drinking water supply network illegally used for irrigation in Tilouline.

agricole local est importante, quoique la récolte et le traitement restent traditionnels et artisanaux» (Touzi et Merzaia-Blama, 2008, p. 269). La commercialisation se fait généralement par les Touaregs qui acheminent le tabac des lieux de production jusqu'à Tamanrasset à proximité de la frontière avec le Niger.

Si l'agriculture moderne peut puiser ses besoins en eau dans la nappe albienne grâce aux forages, l'agriculture oasienne ne possède pas cet avantage. L'eau utilisée est acheminée par des foggaras dont le débit est loin de satisfaire toutes les surfaces irriguées. Afin de combler le déficit en eau d'irrigation, les habitants des ksour ont parfois recours à la réhabilitation de la foggara en l'alimentant par un forage ou par le détournement de l'eau potable. Sachant que les ménages ne payent qu'un forfait de $1500 \mathrm{DA}(11 €)$ pour l'alimentation en eau potable, les agriculteurs oasiens utilisent cette eau quasi gratuite en installant de longues conduites de leurs maisons jusqu'aux jardins. (Fig. 5). Comme le réseau de tuyaux est apparent, les institutions locales tolèrent ce «piratage illégal» de l'eau potable tant que cette eau est destinée à l'activité agricole et donc à améliorer les revenus de la population. Ce phénomène est très répandu dans les ksour du Touat, il l'est moins dans le Gourara et le Tidikelt.

\section{Conclusion}

Le cliché du recul de l'agriculture oasienne au profit d'une agriculture moderne domine dans les instances politiques, sociales et scientifiques depuis une trentaine d'années. Cependant, les nouvelles tendances de l'agriculture saharienne à Adrar devraient amener à infléchir ce discours, comme le montrent aussi Idda et al. (2017). En effet, au moment où la mise en valeur moderne s'oriente ces dernières années vers des cultures industrielles (comme le concombre destiné à la confection de produits cosmétiques), l'agriculture oasienne se dirige vers des cultures plus spécialisées comme le tabac. Ces évolutions des agricultures sahariennes, traditionnelles et modernes, sont des adaptations au contexte politique et économique du pays, caractérisé par le désengagement de l'État et la saturation du secteur tertiaire. Les Oasiens et certains agriculteurs du nord du pays se sont adaptés à cette nouvelle situation en investissant dans l'agriculture saharienne, en profitant de la demande pour certains produits agricoles. La période actuelle est ainsi caractérisée, non par une concurrence entre l'agriculture moderne et l'agriculture oasienne, mais par une recherche commune du profit. Bien que cette relance du secteur agricole ne touche pas toutes les oasis de la région, ses conséquences spatiales sont déjà bien visibles dans l'occupation du sol; il en est de même à Biskra (Amichi et al., 2015). Appréhender son impact économique et social demandera cependant plus d'investigations, sur un terrain qui ne cesse d'évoluer.

\section{Références}

Amichi F, Bouarfa S, Lejars C, Kuper M, Hartani T, Daoudi A, et al. 2015. Des serres et des hommes: des exploitations motrices de l'expansion territoriale et de l'ascension socioprofessionnelle sur un front pionnier de l'agriculture saharienne en Algérie. Cah Agric 24: 11-19. DOI: 10.1684/agr.2015.0736.

Bellal SA, Hadeid M, Ghodbani T, Dari O. 2016. Accès à l'eau souterraine et transformations de l'espace oasien: le cas d'Adrar (Sahara du Sud-ouest algérien). Cahiers de Géographie du Québec 60(169): 29-56.

Bendjelid A, Dari O, Hadeid M, Bellal SA, Gacem F, Belmahi MN. 1999. Mutations sociales et adaptation d'une paysannerie ksourienne du Touat: Ouled Hadj Mamoun (wilaya d'Adrar, Algérie). Insaniyat 7: 39-53.

Bessaoud O. 2008. L'agriculture et la paysannerie en Algérie, les grands handicaps. In: Benghabrit $\mathrm{N}$, Haddab M (eds). L'Algérie 50 ans après, état des savoirs en sciences sociales et humaines, 1954-2004. Oran : CRASC, pp. 359-384.

Bisson J. 2003. Le Sahara: mythes et réalités d'un désert convoité. Paris : L'Harmattan, 479 p.

Capot-Rey R. 1953. Le Sahara français. Paris : PUF, 564 p.

CENEAP. 1990. Plan de développement et d'aménagement de la wilaya d'Adrar - Rapport diagnostic. Alger: CENEAP, 286 p.

Côte M. 2002. Des oasis aux zones de mise en valeur: l'étonnant renouveau de l'agriculture saharienne. Méditerranée 99: 5-14.

Daoudi A, Lejars C, Benouniche N. 2017. La gouvernance de l'eau souterraine dans le Sahara algérien : enjeux, cadre légal et pratiques locales. Cah Agric 26: 35004. DOI: 10.1051/cagri/2017021.

DPAT (Direction de la planification et de l'aménagement du territoire) d'Adrar. 2012. Monographie de la wilaya d'Adrar, 82 p.

Dubost D. 1991. Le blé du Sahara peut-il contribuer à l'autosuffisance de l'Algérie? Bulletin de l'Association de Géographes Français 4: 311-320.

Hadeid M. 2011. La politique de mise en valeur agricole en milieu steppique algérien : un essai de bilan dans les Hautes Plaines sudoranaises (Algérie). Insaniyat 51-52: 99-118.

Hamamouche MF, Kuper M, Lejars C. 2015. Émancipation des jeunes des oasis du Sahara algérien par le déverrouillage de l'accès à la terre et à l'eau. Cah Agric 24: 412-419. DOI: 10.1684/ agr.2015.0777.

Idda S, Bonté B, Mansour H, Bellal SA, Kuper M. 2017. Monument historique ou système bien vivant? Les foggaras des oasis du Touat (Algérie) et leur réalimentation en eau par pompage. Cah Agric 26: 55007. DOI: 10.1051/cagri/2017049.

Lévy J, Lussault M. 2003. Dictionnaire de la Géographie et de l'espace des sociétés. Paris: Belin, 1127 p. 
Marouf N. 1980. Lecture de l'espace oasien. Paris: Sindbad, 281 p. Naouri M, Hartani T, Kuper M. 2015. Mobilités des jeunes ruraux pour intégrer les nouvelles agricultures sahariennes (Biskra, Algérie). Cah Agric 24: 379-386. DOI: 10.1684/agr.2015.0778.

Sahli Z. 1997. Deux tentatives controversées de modernisation de l'agriculture en zone aride : l'opération "tomate d'Adrar" et la mise en valeur hydro-agricole du Touat Gourara (Wilaya d'AdrarAlgérie). Options Méditerranéennes 29(A): 283-295.

Otmane T. 2010. Mise en valeur agricole et dynamiques rurales dans le Touat, le Gourara et le Tidikelt (Sahara algérien). Thèse de doctorat, Universités d'Oran et de Franche-Comté, 400 p.
Ouendeno ML, Daoudi A, Colin JP, 2015. Les trajectoires professionnelles des jeunes dans la néo-agriculture saharienne (Biskra, Algérie) revisitées par la théorie de l'agricultural ladder. Cah Agric 24: 396-403. DOI: 10.1684/agr.2015.0793.

Ould Rebai A, Hartani T, Chabaca MN, Kuper M. 2017. Une innovation incrémentielle : la conception et la diffusion d'un pivot d'irrigation artisanal dans le Souf (Sahara algérien). Cah Agric 26: 35005. DOI: $10.1051 /$ cagri/2017024.

Touzi A, Merzaia-Blama A. 2008. La conservation des denrées agroalimentaires par séchage dans les régions sahariennes. Revue des Energies Renouvelables, SMSTS(8): 267-272.

Citation de l'article : Hadeid M, Bellal SA, Ghodbani T, Dari O. 2018. L'agriculture au Sahara du sud-ouest algérien : entre développement agricole moderne et permanences de l'agriculture oasienne traditionnelle. Cah. Agric. 27: 15005. 